Article

\title{
A Power Coupling System for Electric Tracked Vehicles during High-Speed Steering with Optimization-Based Torque Distribution Control
}

\author{
Hong Huang ${ }^{1,2}$, Li Zhai ${ }^{1,2, *(\mathbb{D})}$ and Zeda Wang ${ }^{1,2}$ \\ 1 National Engineering Laboratory for Electric Vehicles, Beijing Institute of Technology, Beijing 100081, China; \\ 2120160333@bit.edu.cn (H.H.); wzd950628@163.com (Z.W.) \\ 2 Co-Innovation Center of Electric Vehicles in Beijing, Beijing Institute of Technology, Beijing 100081, China \\ * Correspondence: zhaili26@bit.edu.cn; Tel.: +86-010-6891-5202
}

Received: 19 April 2018; Accepted: 11 June 2018; Published: 13 June 2018

check for updates

\begin{abstract}
It is significant to improve the steering maneuverability of dual-motor drive tracked vehicles (2MDTVs), which have wide applications in the tracked vehicle industry. In this paper, we focus on the problem of insufficient propulsion motor power during high-speed steering. Some correction formulas are introduced to improve the accuracy of the mathematical model. A steering coupling system and an optimization-based torque distribution control strategy is adopted to improve the lateral stability of the vehicle. The 2MDTV model and the proposed control strategy are built in the multi-body software RecurDyn and the control software Matlab/Simulink, respectively. According to the real-time steering simulation by the hardware-in-the-loop (HIL) method, the 2MDTV with the coupling device outputs more power during high-speed steering. The results show the speed during steering is quite high though, the stability of the vehicle can be achieved due to using the torque distribution strategy, and the steering maneuverability of the vehicle is also improved.
\end{abstract}

Keywords: tracked vehicle; dual-motor; high-speed steering; power coupling; torque control

\section{Introduction}

With the development of modern electronic technology, traditional mechanical transmissions may possibly be replaced by electric drive systems due to the application of advanced electrical equipment like high power density motors and power inverters. Electric drive systems are used not only in wheeled vehicles, but also in tracked vehicles. Electric tracked vehicles (ETVs) can operate at any steering radius and achieve continuously variable speed regulation, which avoids the mechanical vibration caused by the transmission shift. Moreover the layout of the electric drive system is more flexible and it's easy to achieve modular management in ETVs. It can be predicted that the electric drive technology is a major trend in the development of ETV power transmission systems for military, agriculture, construction and mining applications [1].

Dual-motor independent drives are generally used in the electric drive systems of ETVs [2]. By controlling the speed or torque of the traction motor on both sides to achieve the coordinated control of the longitudinal and lateral forces, respectively, the ETV can drive straight or turn with different radii [3]. As a result of the dual-motor independent drive structure, the steering and transmission mechanism found in traditional tracked vehicles is unnecessary. By real-time control of the speed or torque of the traction motor on both sides, the vehicle can achieve continuously variable steering, which is called electronic control differential steering (ECDS). This comprehensive electronic differential steering control can significantly improve the steering stability and trajectory tracking [4].

Different from wheeled vehicles, skid steering is widely used in tracked vehicles. Therefore, due to the contact between the track and the ground, there is a steering resistance moment under the 
steering situation. In particular, when the 2MDTV turns at low speed with small radius, the vehicle will be subjected to a large steering resistance moment, so the motor torque required is higher than the vehicle's demand estimated based on straight running condition [5]. In addition, when the vehicle turns at high speed, the motor power required for the outer side track is higher, which results in the need for a high power motor with high overload capacity, leading to larger size of the motor and power electronic device. It is worth noting that in the dynamic steering, the requirements of motor torque and power are also much higher than that in the steady state steering [4].

Thus, based on the above challenges, many power coupling devices have been proposed to reduce the power requirements for a single track have been designed and added between two traction motors to couple the power of the two motors [6-8]. However, the motors cannot be controlled well enough to realize infinitely variable steering because of the constraint of a shaft between the two motors. Gai et al. [9] also proposed a coupling transmission arrangement to achieve power coupling, so that the power regenerated from the sprocket in low-speed side can flow to the sprocket in the high-speed side. However, due to the use of four planetary gear units in the coupling transmission arrangement, this system is very complicated and bulky. Moreover it is difficult to obtain better steering radius following electronic differential steering. A half shaft differential control structure [10] was proposed to allow simple coupling of power from the two half shafts and control of the relative speed between the two half shafts. However it increases the size of the assembly system. The power-split-based transmission scheme and coupling characteristics of the planet coupled device were analyzed [11]. The electro-mechanical transmission is composed of an engine, two motors, a planet coupling device and a planetary transmission. The power from the engine and the motor can be coupled by the planet coupling device, thereby increasing the output power of the vehicle. However planet couple devices are used in wheeled vehicles and are not suitable for tracked vehicles. A power coupling assembly based on fixed shaft coupling is proposed to couple the torque from a main motor and an auxiliary motor [12]. The output torque of the outside sprocket increases with the coupling assembly, but the scheme with two main motors and two auxiliary motors does not reduce the total power requirements for motors in 2MDTVs. A steering coupling system composed of a steering motor, two planetary gear couplers and two propulsion motors is designed to improve the output power [13]. However the mechanical characteristics of the coupler and the steering performance during high speed were not fully considered.

The steering control of tracked vehicles can be divided into two categories generally: adjusting speed difference between two sprockets based on speed or torque control [14,15]. A coordinated hierarchy control strategy of driving torque is proposed for distributed electric drive tracked vehicles [16]. The quadratic programming method is used to design the torque optimization distribution law of the in-wheel motors. A steering control strategy has been proposed for tracked vehicles propelled by electric transmissions [17]. The self-adaptive control strategy is designed on the basis of Model Reference Adaptive Control Theory. This strategy could be used to effectively adjust the motors' driving torques and ensure great steering stability. Some research focus on the energy management strategies of the hybrid electric powertrain to improve the fuel economy [18]. Algorithms such as Stochastic Dynamic Programming, Radau Pseudospectral Method and Reinforcement Learning are implemented for optimizing the energy management strategy [19-21].

However, few studies have focused on the maneuverability and stability of the 2MDTV during high speed steering [22]. Most of the existing analyses investigate the tracked vehicles without coupling devices during low-speed steering, so the proposed theory and control strategies are only adapted to low-speed steering, and have some limitations for high-speed steering. Due to the absence of the stability control and the shortage of propulsion motor power, the problem of torque allocation for 2MDTVs during steering is still unsolved. In addition, tracks is prone to slip considering the high speed of the vehicle and the adhesion coefficient of the road. At the same time the vehicle is more sensitive to the outside interference caused by the surroundings and pavements, so the vehicle may be out of speed or even roll over if the control system cannot meet the requirements. 
In this paper, the impact of efficiency decline is taken into consideration and an electromechanical coupling device is proposed to solve the problem of insufficient power during high-speed steering in curves of different radii. An optimal torque distribution strategy based on torque control considering the slip between track and pavement is proposed to achieve better torque and power utilization. The steering co-simulation model is built by the multi-body software Recurdyn and the control software Matlab/Simulink. A real-time electronic steering control simulation by the Hardware-in-the-Loop (HIL) method verifies the effectiveness of the coupling device and the proposed strategy.

\section{Mathematical Model for Steering}

The steering of tracked vehicled is achieved by adjusting the speed difference between the two tracks. The electric drive structures of the electric tracked vehicle includes dual-motor independent drive, single motor drive, and hybrid electromechanical complex drive. Dual-motor independent drive structures have been widely used due to their simple structure, high transmission efficiency, flexible layout and other advantages. In this paper, a dual-motor independent drive electric tracked vehicle (2MIETV) is selected, and the vehicle's configuration is as shown in Table 1.

Table 1. Vehicle Parameters.

\begin{tabular}{cccc}
\hline Parameters & Value & Parameters & Value \\
\hline Vehicle tread, $B(\mathrm{~m})$ & 1.3 & Drive ratio, $i_{g}$ & 6.35 \\
Ground contact length, $L(\mathrm{~m})$ & 1.7 & Mass of vehicle, $m(\mathrm{~kg})$ & 2800 \\
Rolling resistance coefficient, $f$ & 0.04 & Mass gain coefficient, $\delta$ & 1.5 \\
Transmission efficiency, $\eta$ & 0.9 & Moment of inertia, $J\left(\mathrm{~kg} / \mathrm{m}^{2}\right)$ & 3000 \\
\hline
\end{tabular}

The vehicle dynamics performance indicators during straight running are listed as follows:

(1) Maximum speed should be $72 \mathrm{~km} / \mathrm{h}$.

(2) Maximum climbing degree should be $32^{\circ}$ and the climbing speed is $18 \mathrm{~km} / \mathrm{h}$.

(3) Acceleration capability: 0 to $32 \mathrm{~km} / \mathrm{h}$ in less than $8 \mathrm{~s}$.

The parameters of the propulsion motor can be determined by the above indicators in general [23], where the transmission efficiency is assumed as a constant value of 0.9. However, the transmission efficiency will decrease while the vehicle speed increases due to the friction loss of crawler mechanism consisting of tracks, sprockets, road wheels, inducers, etc. Then the efficiency of transmission system during the high-speed running can be defined as follows [24]:

$$
\eta_{t}=0.95-0.0017 v(v>30 \mathrm{~km} / \mathrm{h})
$$

where $\eta_{t}$ is the transmission efficiency, $v$ is vehicle speed.

In addition, the loss due to internal resistance in crawler mechanism of tracked vehicle cannot be ignored during high-speed driving, and the power consumed by internal resistance in electric tracked vehicles is defined as follows [25]:

$$
P_{i r}=\frac{0.46 m g v}{7200000}\left(30+0.9 \frac{v}{1.6}\right)
$$

where $P_{i r}$ is the power consumed by internal resistance in crawler mechanism.

The above power loss described in Equation (2) was not considered in a previous study [23], and since the output power of propulsion motor cannot satisfy the requirements for high-speed running, the parameters of the drive motor should be determined by consideration of $P_{i r}$ at high running, as shown in Table 2. 
Table 2. Motor Parameters Comparison.

\begin{tabular}{cccc}
\hline Parameters & Rated/Maximum Power & Rated/Maximum Torque & Rated/Maximum Speed \\
\hline Loss considered & $32 / 50 \mathrm{~kW}$ & $122 / 215 \mathrm{~N} \cdot \mathrm{m}$ & $2500 / 9000 \mathrm{rpm}$ \\
Loss ignored & $20 / 40 \mathrm{~kW}$ & $64 / 150 \mathrm{~N} \cdot \mathrm{m}$ & $3000 / 9000 \mathrm{rpm}$ \\
\hline
\end{tabular}

We took right steering as an example, $F_{L}$ is the traction force on the outer track, $F_{R}$ is the tractionforce on the inner track, $R_{L}$ is the rolling resistance force on the outer track, $R_{R}$ is the rolling resistance on the inner track, $M_{\mu}$ is the steering resistance moment, $v_{L}$ is the track speed on the outer side, $v_{R}$ is the track speed on the inner side and $\omega$ is the yaw rate. Steering maneuvers with different radii can be shown in Figure $1 . M_{\mu}$ is defined as follows [26]:

$$
M_{\mu}=\frac{1}{4} \mu m g L=\frac{1}{4} \frac{\mu_{\max }}{0.925+0.15 \cdot \frac{R}{B}} m g L
$$

where $\mu$ is the coefficient of roll resistance, $\mu_{\max }$ is the maximum rolling resistance coefficient, $R$ is the steering radius of the vehicle. The rolling resistance of the two tracks is the same in magnitude but opposite in direction when $v_{R}$ and $v_{L}$ are opposite in sign. If $v_{R}$ and $v_{L}$ are same in sign, the rolling resistance of the two tracks will be the same in direction. The rolling resistance $R_{L}$ and $R_{R}$ are expressed as follows:

$$
R_{L}=R_{R}=\frac{1}{2} f m g
$$

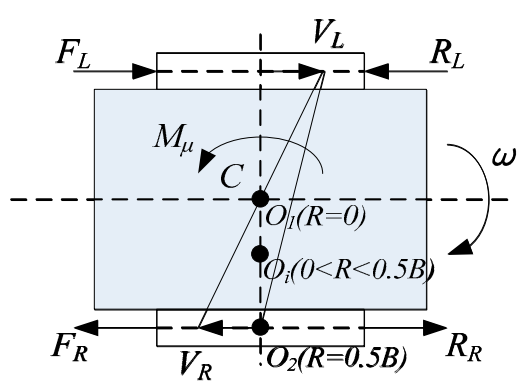

(a)

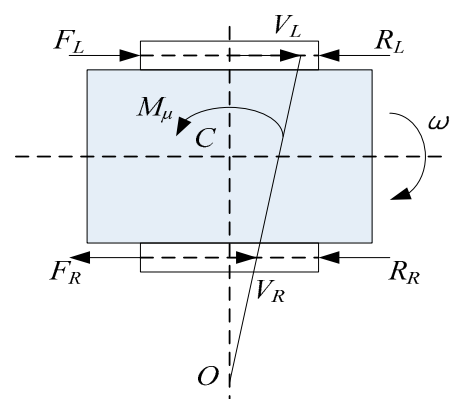

(b)

Figure 1. Forces analysis (a) $R \leq 0.5 B$ steering; (b) $R>0.5 B$ steering.

\subsection{Small Radius Steering $(R \leq 0.5 B)$}

This situation can be further divided into three categories: $R=0,0<R<0.5 B, R=0.5 B$. The equilibrium relationship between the force and moment of the vehicle when turning from resting, as shown in Figure 1a, can be expressed as:

$$
\left\{\begin{array}{l}
F_{L}-F_{R}+R_{R}-R_{L}=\delta m \frac{d v}{d t} \\
\left(F_{L}+F_{R}\right) \cdot \frac{B}{2}-\left(R_{L}+R_{R}\right) \cdot \frac{B}{2}-M_{\mu}=J \frac{d \omega}{d t}
\end{array}\right.
$$

where $F_{L}$ and $F_{R}$ are tractive force but opposite in direction.

It' worth mentioning that when $R=0, F_{L}=F_{R}, V_{L}=V_{R}$, lateral acceleration is zero, $d v / d t=0$, and when $R=0.5 B, V_{R}=0, R_{R}=0$.

The motor speed on both sides can be expressed as:

$$
\left\{\begin{array}{l}
n_{L}=\frac{1000 i_{g}}{120 \pi r_{z}} \cdot 3.6 \cdot \omega \cdot\left(\frac{B}{2}-R\right) \\
n_{R}=\frac{1000 i_{g}}{120 \pi r_{z}} \cdot 3.6 \cdot \omega \cdot\left(\frac{B}{2}+R\right)
\end{array}\right.
$$


where $r_{z}$ is the radius of the sprocket, $r_{z}=150 \mathrm{~mm}$

From (1)-(6), the motor torque and power can be obtained as follows:

$$
\left\{\begin{array}{l}
T_{L, R}=\frac{F_{L, R}}{i_{g} \eta_{t}} \cdot r_{z} \\
P_{L, R}=\frac{T_{L, R} \cdot n_{L, R}}{9549}
\end{array}\right.
$$

$T$ is tractive torque but opposite in direction. $P$ is consumed power used to drive the track. Subscript " $L$ " or " $R$ " means the left or right side of the vehicle.

\subsection{Big Radius Steering $(R>0.5 B)$}

According to the vehicle dynamic balance, the following formula can be obtained from Figure 1b:

$$
\left\{\begin{array}{l}
F_{L}-F_{R}-R_{R}-R_{L}=\delta m \frac{d v}{d t} \\
\left(F_{L}+F_{R}\right) \cdot \frac{B}{2}+\left(R_{R}-R_{L}\right) \cdot \frac{B}{2}-M_{\mu}=J \frac{d \omega}{d t}
\end{array}\right.
$$

where $F_{L}$ is tractive force. In steady-state steering conditions, the expressions of the driving force can be obtained and if we assume the $F_{R}$ is null:

$$
F_{R}=-\frac{1}{2} f m g+\frac{\mu m g L}{4 B}=-\frac{1}{2} f m g+\frac{\mu_{\max } m g L}{3.7 B+0.6 R}=0
$$

We thus obtain the solution $R=R_{0}=67.54 \mathrm{~m}$.

This situation can be also divided into three categories: $0.5 B<R<R_{0}, R=R_{0}, R>R_{0}$. When $R>R_{0}, F_{R}$ is traction force and is in the same direction with the track. When $R=R_{0}, F_{R}=0$, $P_{R}=0$. At this moment, the inner propulsion motor is turned off, the track rotates freely, only the outer propulsion motor works. While when $R<R_{0}, F_{R}$ is brake force, generated by motor. The direction between $F_{R}$ and $V_{R}$ is opposite in this situation.

Similarly, during high-speed steering the internal resistance in crawler mechanism increases, so the decline of transmission efficiency cannot be ignored. Therefore, a factor $k$ is proposed to correct Equation (1). The value of $k$, which is determined by simulation or experimental results, ranges from 0.0001 to 0.002 :

$$
\eta_{t}=0.95-(0.0017+k) v(v>30 \mathrm{~km} / \mathrm{h})
$$

\subsection{Calculation}

The steering process can be divided into two periods: dynamic steering and static steering, the motor torque and power required vary with the steering radius and speed. This paper focuses on the steering performance of the vehicle at high speeds. Usually, the yaw rate of ETV is $45 \sim 75^{\circ} / \mathrm{s}$, we choose $w=1.3 \mathrm{rad} / \mathrm{s}$. During high-speed steering, in order to prevent the crew from feeling discomfort due to the excessive centripetal acceleration, the centripetal acceleration is limited to $1 \mathrm{~g}$. According to the calculation results, several representative cases are selected, as shown in Table 3.

It can be seen from Table 3 that during low-speed small radius steering, the demand torque of the vehicle is large due to the large steering resistance moment, which exceeds the maximum torque that the motor can provide. This has been confirmed in the previous study. 
Table 3. Torque and power required by two motors in stationary steering.

\begin{tabular}{|c|c|c|c|c|c|c|c|c|c|}
\hline $\begin{array}{c}R \\
(\mathrm{~mm})\end{array}$ & $\begin{array}{c}T_{L} \\
(\mathrm{~N} \cdot \mathrm{m})\end{array}$ & $\begin{array}{c}T_{R} \\
(\mathbf{N} \cdot \mathbf{m})\end{array}$ & $\begin{array}{c}n_{L} \\
(\mathrm{r} / \mathrm{min})\end{array}$ & $\begin{array}{c}n_{L} \\
(\mathrm{r} / \mathrm{min})\end{array}$ & $\begin{array}{l}P_{L}^{\prime}{ }^{1} \\
(\mathrm{~kW})\end{array}$ & $\begin{array}{c}P_{L}{ }^{2} \\
(\mathrm{~kW})\end{array}$ & $\begin{array}{c}P_{R} \\
(\mathbf{k W})\end{array}$ & $\begin{array}{c}V \\
(\mathrm{~km} / \mathrm{h})\end{array}$ & $\begin{array}{c}w \\
(\mathrm{rad} / \mathrm{s})\end{array}$ \\
\hline$O B$ & 278.7 & -278.7 & 344 & -344 & 10.0 & 10.0 & 10.0 & 0 & 1.3 \\
\hline $2 B$ & $\overline{214.9}$ & $\overline{-178.1}$ & 1720 & 1032 & 38.7 & 38.7 & -19.2 & 13.0 & 1.3 \\
\hline $5 B$ & 162.1 & -125.3 & 3549 & 2904 & $\underline{60.3}$ & $\underline{60.3}$ & -38.1 & 28.8 & 1.2 \\
\hline $10 B$ & 117.7 & -80.9 & 4791 & 4335 & $\overline{59.0}$ & $\overline{64.2}$ & -36.7 & 40.7 & 0.87 \\
\hline $20 B$ & 84.2 & -45.3 & 6295 & 6618 & $\overline{55.3}$ & $\overline{64.8}$ & -29.9 & 57.5 & 0.6 \\
\hline $30 B$ & 68.0 & -28.1 & 7992 & 7730 & $\overline{52.9}$ & $\overline{66.0}$ & -22.8 & 70.4 & 0.5 \\
\hline
\end{tabular}

${ }^{1} P_{L}^{\prime}$ : required power (internal loss ignored). ${ }^{2} P_{L}$ : required power (internal loss considered).

During high-speed steering, the vehicle's power requirements $(60.3 / 64.2 / 64.8 / 66 \mathrm{~kW})$ exceed the maximum power $(50 \mathrm{~kW})$ that the motor can provide, so two motors with at least $70 \mathrm{~kW}$ are required for a vehicle without a coupling device, whereas during straight driving the required power is only half of the motor maximum power [4]. If the arrangement with coupling device $(50+50+20 \mathrm{~kW})$ is applied, the peak power will reduce to $20 \mathrm{~kW}$ less than the arrangement with two independent propulsion motors $(70+70 \mathrm{~kW})$. It is noteworthy that the values shown in Table 3 are calculated with $k=0$. If $k \neq 0$, the lateral power demand can be determined by Equation (11). As $k$ increases, the power demand actually for the outside motor will be further increased:

$$
P_{L}=\left\{\begin{array}{l}
\frac{53.17}{-40.65 k+0.8809}+3.843(R=10 B) \\
\frac{49.74}{-57.49 k+0.8523}+6.397(R=20 B) \\
\frac{41.2}{-60.98 k+0.8463}+8.741(R=30 B)
\end{array}\right.
$$

\section{Steering System Design}

\subsection{Steering Coupling Drive System}

With the deepening of research and advances in power electronics technology, the AC-DC-AC electric transmission structure is mainly used in electric drive systems. The unilateral (propulsion) motor is mainly an induction motor or permanent magnet motor with reliable control technology [27]. In general, the engine-generator generates energy, and the energy is allocated to the propulsion motors through the energy conversion unit. According to the control strategy, the speed, torque, power and other parameters of the motor is adjusted by the motor controller to drive the sprocket. In the regenerative braking mode, the energy regenerated from the wheel is distributed by the energy conversion and distribution unit, partially or completely to the energy battery pack. And the rest part of the energy flows to the outside track or energy absorb unit, thereby improving the energy utilization and stability of the vehicle.

The steering coupling drive system is composed of a new type of center steering motor, two electromagnetic clutches, two planetary gear couplers, and two propulsion motors, as shown in Figure 2. The steering motor is smaller with lower rated power $10 \mathrm{~kW}$ (maximum power $20 \mathrm{~kW}$ ). A reverse mechanism is designed in the steering motor to achieve positive and negative rotation direction. When the torque or power is insufficient, the electromagnetic clutch is engaged and the torque or power of the driving wheel is satisfied by the coupling between the steering motor and the propulsion motor. 


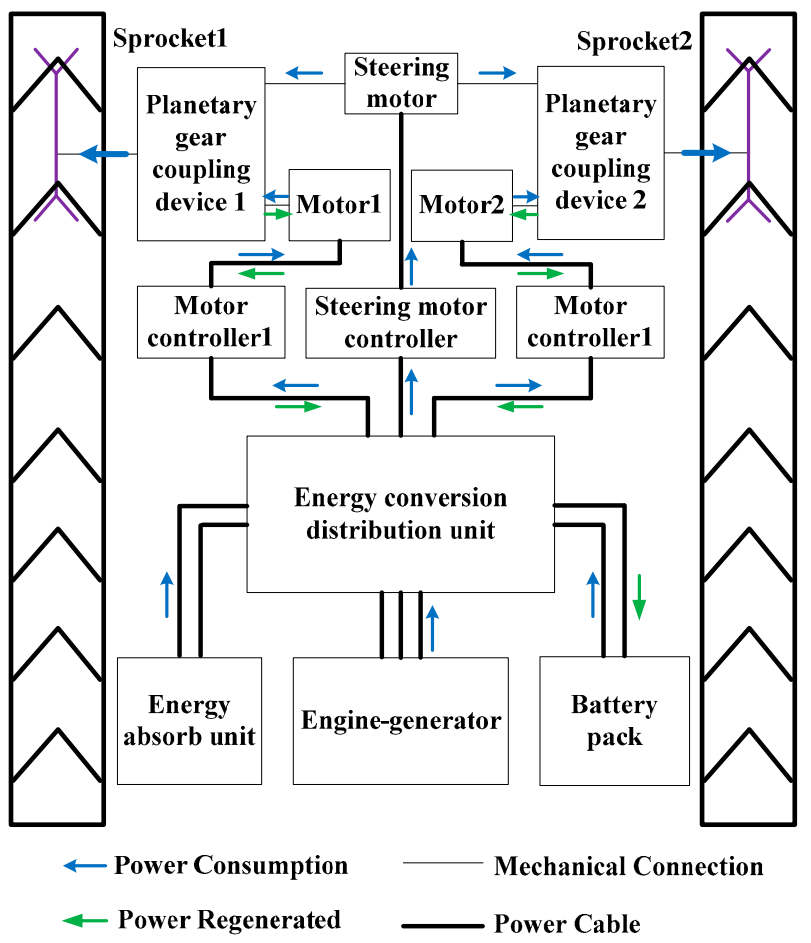

Figure 2. Steering coupling drive system configuration and power flow.

\subsection{Planetary Gear Coupler Design}

The planetary gear couplers consists of two electromagnetic (EM) clutches, two gear pairs and a planetary gear unit, which consists of a sun gear, a ring gear, a planet carrier and three planet gears, as shown in Figure 3. Gear pair 1 contains gear 1 and gear 2 (ring gear), whereas gear pair 2 contains gear 3 and gear 4 . The driving disc of electromagnetic clutch is connected with steering motor shaft, while clutch plate is connected with gear. It's worth noting that gear 1 and gear 3 are mounted on the steering motor shaft without a spline, transmitting the power only when one of the EM clutches is engaged.

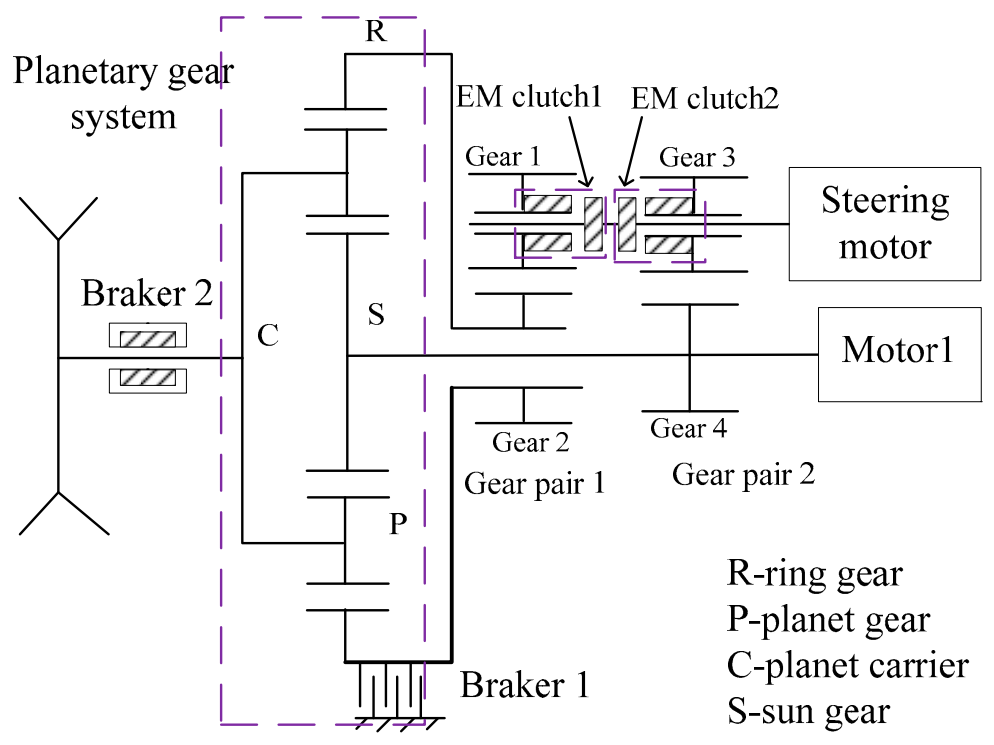

Figure 3. Components of the planetary gear coupler. 
When the vehicle is driving straight, the ring gear $(\mathrm{R})$ is fixed by brake 1 while both the EM clutch 1 and the EM clutch 2 are disengaged. The power is transmitted to the sun gear (S) of the planetary gear unit (transmission ratio 6.35) from the propulsion motor, and outputted to the sprocket through the planet carrier(C), as shown in Figure 4a. The device works in different modes according to different steering conditions. Figure $4 \mathrm{~b}-\mathrm{d}$ shows the power transmission routes during some typical steering conditions.

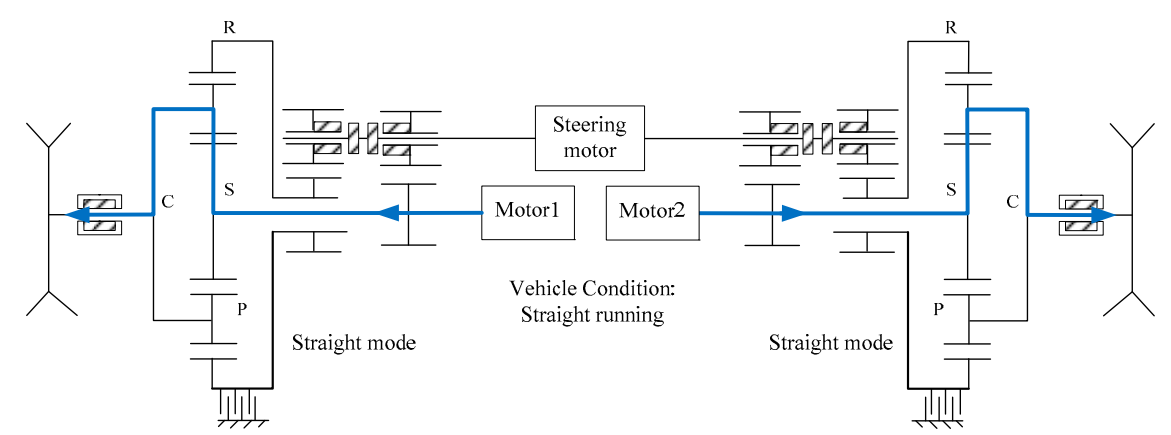

(a)

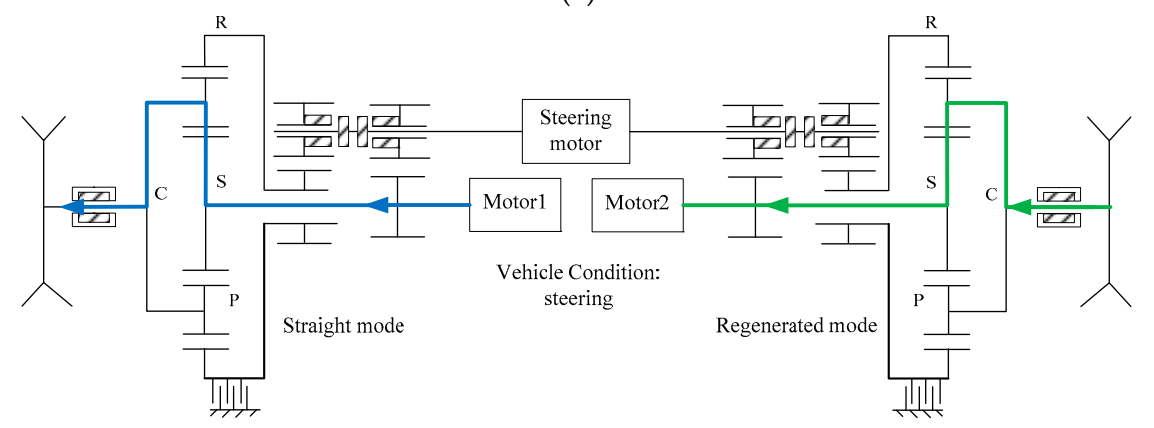

(b)

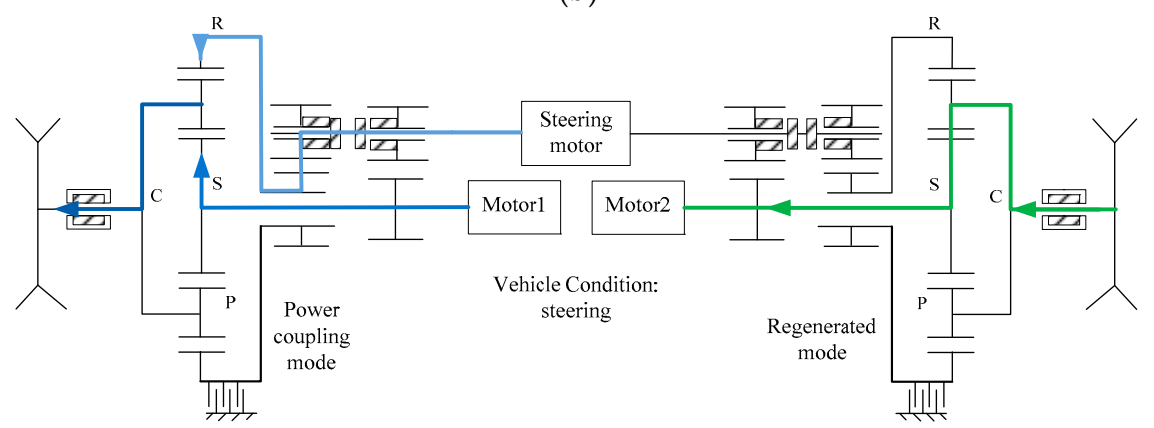

(c)

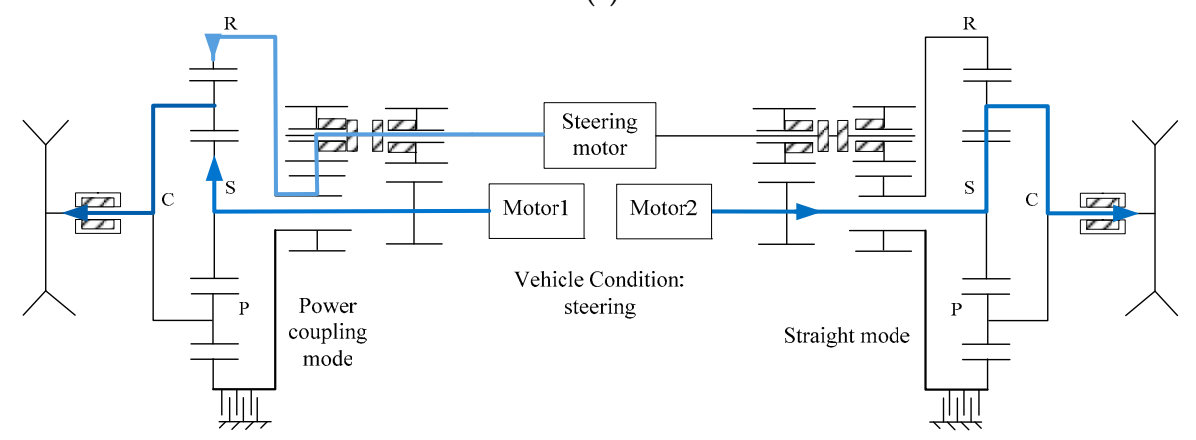

(d)

Figure 4. (a) Transmission mode during straight running; (b) Transmission mode during low speed steering; (c) Transmission mode during medium speed steering; (d) Transmission mode during high speed steering. 
During low-speed steering, if the torque demand for the sprocket is too large, the torque coupling mode is active. The torque of the steering motor and the propulsion motor is coupled via the gear pair 2, and this drives the sprocket through the planet carrier. This mode was discussed in previous studies [22]. If the torque demand does not exceed the peak torque of the propulsion motor, the steering motor is turned off and the EM clutches are disconnected. The outer propulsion motor provides the required torque for steering, while the inner motor is in the regenerative braking mode, converting the mechanical energy from the crawler into electrical energy. The power transmission path is shown in Figure $4 b$.

The power demand for the outer sprocket may be larger during medium-speed steering. If it exceeds the maximum power of the propulsion motor, the steering motor works and the device works in the power coupling mode. The EM clutch 1 is engaged while the EM clutch 2 is disconnected and the brake 1 is disengaged. The power of the steering motor is transferred to ring gear $(\mathrm{R})$ through the gear pair1, and the power of propulsion motor is transmitted to the sun gear (S). Then the power is coupled and transmitted to the sprocket through the planet carrier (C). In this case, the inner motor is still in regenerative braking mode. The corresponding power transmission route is shown in Figure 4c.

During high-speed steering, the propulsion motors on both sides are in the drive mode for high-speed steering. The outer coupling device is in power coupling mode due to the high power requirements for the outer track, and the steering motor provides auxiliary power to keep the vehicle steering at high speed. The inner motor also outputs power to maintain the vehicle speed. The power transmission route is shown in Figure $4 \mathrm{~d}$.

In general, the device works in torque coupling mode during low speed steering and in power coupling mode during high speed steering. The operation of components in different coupling modes is listed in Table 4. In this paper, we focused more on high speed steering while the low speed steering had been discussed yet [22,23].

Table 4. Coupling mode and the operation of each component.

\begin{tabular}{cccccc}
\hline Mode & Brake 1 & EM Clutch 1 & EM Clutch 2 & Steering Motor & Propulsion Motor \\
\hline straight running & engaged & disconnected & disconnected & off & working \\
torque coupling & engaged & disconnected & combined & working & working \\
power coupling & disengaged & combined & disconnected & working & working \\
\hline
\end{tabular}

\section{Control Strategy}

A closed-loop control strategy based on torque control of motor is proposed and shown in Figure 5. The strategy proposed for a 2MDTV is designed as a three-level structure, including an ideal model level, a dynamics upper-level controller and a torque distribution lower-level controller. In the reference model level, a dynamic reference model is established and discussed in Section 2 to obtain the desired yaw rate or other dynamics parameters, according to the driver inputs and the actual state of the vehicle. The upper-level controller consists of a speed tracking controller and a yaw moment controller with the PID control method and fuzzy PID control, respectively. The controller in this level aims to guarantee the capability of speed following and basic steering performance. The lower-level controller adopts the optimal allocation algorithm to assign the optimal torque $\left(T_{L}\right.$, $\left.T_{R}\right)$ to desired torque $\left(T_{L-d e s}, T_{s-d e s}, T_{R \text {-des }}\right)$ generated by propulsion motors and steering motor under the constraints of the planetary coupling device. Based on the above calculation, the output torque of two propulsion motors and steering motor can be directly distributed to three motor controllers by a CAN bus, respectively. Finally, the torque or power is coupled through the electromechanical coupling device and transmitted to sprockets to improve the stability of the 2MDTV. 


\subsection{Yaw Moment Fuzzy PID Controller}

The yaw moment controller is designed based on the error between the actual angular speed and the desired angular speed to calculate the target yaw moment $M_{z \text {-des }}$ by the fuzzy PID control method. This controller is activated when the yaw rate error $\Delta \omega$ beyond the constant 0.1 .

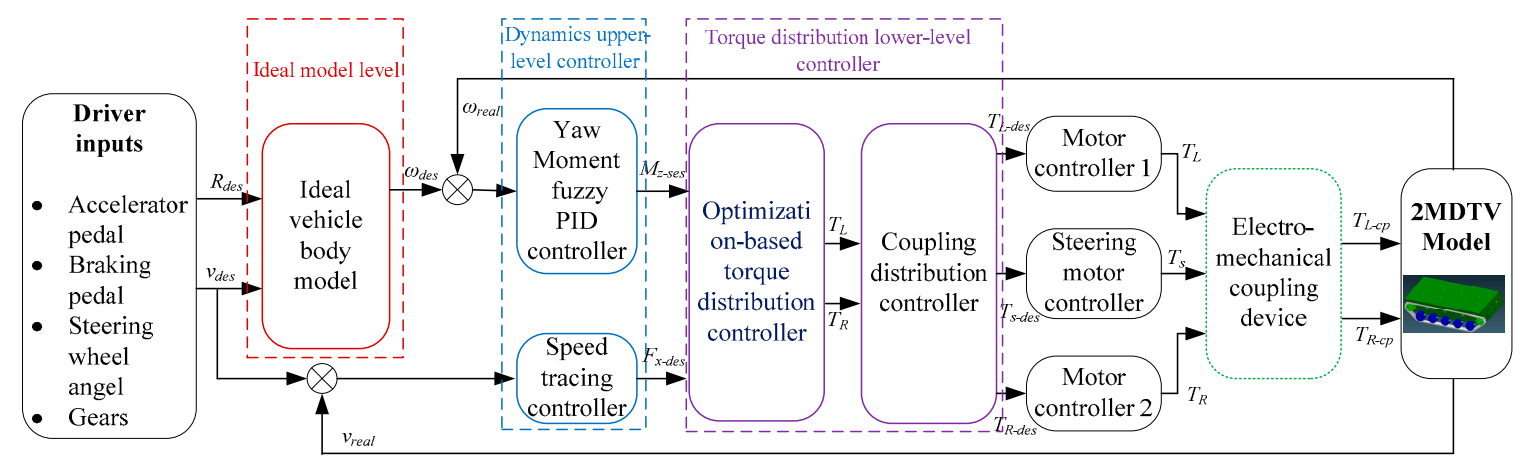

Figure 5. Control strategy schematic based on torque.

\subsection{Speed Tracing Controller}

The speed tracking controller is always activated and designed based on the PID control method to calculate the desired traction force of vehicle $F_{x \text {-des }}$ for the purpose of following the desired vehicle speed. Usually during high speed steering, the speed of the vehicle declines rapidly and the steering mode is translated into low speed steering, so the speed tracking controller is important especially for high speed steering.

\subsection{Optimization-Based Distribution Controller}

To improve the performance of the speed and yaw rate following, an optimization-based distribution controller is designed to obtain the optimal torque $T_{L}$ and $T_{R}$ of the bilateral sprockets. According to Figure 5 , the torque $T_{L}, T_{R}$ and $T_{S}$, which are supposed to be generated by propulsion motors and steering motor, are allocated by the coupling distribution controller under the constraint of planetary coupling to track desired steering radius.

The rolling resistance is assumed to be zero and then the matrix form $H$ can be obtained according to the dynamic balance analysis in Section 2 and expressed as follows:

$$
\kappa=H \cdot u
$$

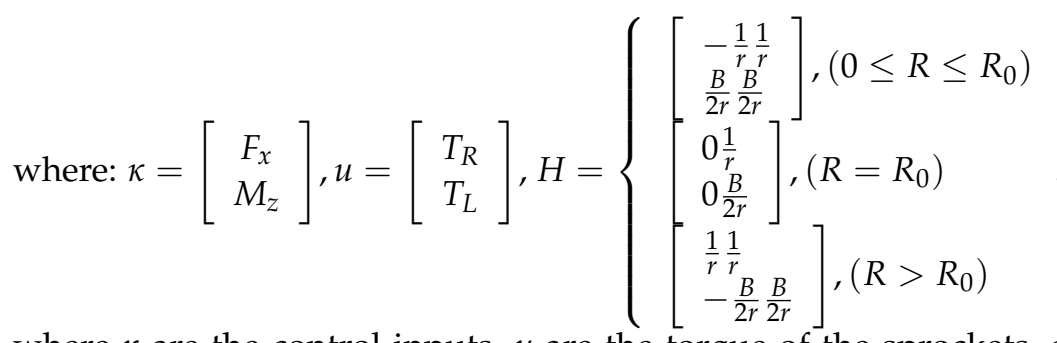

where $\kappa$ are the control inputs, $u$ are the torque of the sprockets, and $H$, the control effectiveness matrix, is chosen depending on the steering radius. The optimization object to minimize is chosen as distribution error, $\|H \times u-\varepsilon\|$, for control accuracy and $u$, for efficient driving. We thus obtain the optimization objective function:

$$
u=\underset{u_{\min } \leq u \leq u_{\max }}{\operatorname{argmin}}\left[\left\|W_{u} \cdot u\right\|^{2}+\xi \cdot\left\|W_{v} \cdot(H \cdot u-\kappa)\right\|^{2}\right]
$$


This is a weighted least-squares (WLS) problem, and where $W_{u}$ is the weighting matrix for efficient driving, $W_{v}$ is the diagonal weighting matrix of the inputs, $\xi$ is the weighting factor whose value is chosen depending on how important the distribution error is. The objective function is determined by the following constraints:

$$
\left\{\begin{array}{l}
\left|T_{L}\right| \leq T_{L, \max },\left|T_{R}\right| \leq T_{R, \max } \\
-T_{\text {max }} \text { brake } \leq T_{L, R, s} \leq T_{\text {max-drive }} \\
\left|T_{L}\right| \leq \frac{\text { frmg }}{2},\left|T_{R}\right| \leq \frac{\text { frmg }}{2} \\
\varepsilon_{m} \geq 90 \%
\end{array}\right.
$$

where $\varepsilon$ is the efficiency of motor obtained by map diagram of speed-torque.

\subsection{Coupling Distribution Controller}

The coupling distribution controller allocate the torque $T_{L}, T_{R}$ and $T_{S}$ according to the torque $T_{L}$ and $T_{R}$ received form the optimization-based distribution controller in different situations. The torque distribution is based on following constraints:

$$
\left\{\begin{array}{l}
-T_{\text {max-brake }} \leq T_{L, R} \leq T_{\text {max-drive }} \\
\left|T_{S}\right| \leq T_{S, \text { max }} \\
T_{\text {sun }}: T_{\text {ring }}: T_{\text {carrier }}=1: i-1: i \\
n_{\text {sun }}+(i-1) n_{\text {ring }}-i n_{\text {carrier }}=0
\end{array}\right.
$$

In order to reduce the complexity of the control system, the priority for dual motor operating mode is the highest. The desired torque $\left(T_{L-d e s}, T_{s-d e s}, T_{R \text {-des }}\right)$ can be obtain according to Equation (15) and coupling judgment module [23]. The steering motor works only when the propulsion motor can't generate enough torque or power during some steering situations.

\section{Modeling and Simulation Results}

We build the steering co-simulation model using the multi-body software Recurdyn and control software Matlab/Simulink. The vehicle model and ground model are developed in Recurdyn, as shown in Figure 6. The driver inputs model, the control strategy model and the motor system model are developed in Simulink. The multi-body dynamic model in Recurdyn is seamlessly interfaced with Matlab/Simulink. The parameters of the dynamic simulation parameters are shown in Table 5.

Table 5. Dynamic simulation parameters.

\begin{tabular}{ccccccc}
\hline \multirow{2}{*}{ Component } & \multirow{2}{*}{ Number } & \multicolumn{3}{c}{ Inertia $\mathbf{( k g \cdot \mathbf { m m } ^ { 2 } \mathbf { ) }}$} & \multirow{2}{*}{ Stiffness Coefficient } & Damping Coefficient \\
\cline { 3 - 5 } & & $\mathbf{I x x}$ & $\mathbf{I y y}$ & $\mathbf{I z z}$ & & 10 \\
\hline Sprocket & 2 & 237,774 & 237,774 & 279,379 & 10,000 & 10 \\
Road Wheel & 10 & 495,799 & 495,799 & 685,898 & 10,000 & 10 \\
Idler & 2 & 495,799 & 495,799 & 685,898 & 10,000 & 10 \\
Carrier & 6 & 16,169 & 16,169 & 5121 & 10,000 & 60 \\
Balance Arm & 10 & 1157 & 3987 & 5056 & $17,319,000$ & 1000 \\
Track link & 198 & 5425 & 5493 & 1989 & $1,000,000$ & \\
\hline
\end{tabular}

The RecurDyn software can define soil parameters according to the user's needs. The road is divide into rectangular elements for calculation. Therefore, the contact force between the track and the ground can be automatically calculated by the software. The calculation is based on the following two models: a hard pavement model established by general contact force, and a soft pavement model based on Baker's theory. We choose the hard pavement model in this paper. 
The pressure between the track shoes and the ground on hard pavement is defined by the track contact. The contact-collision force in RecurDyn is calculated as:

$$
F=-k\left(q-q_{0}\right)^{n}-c q
$$

where $q$ is ground contact position before contact, $q_{0}$ is ground contact position after contact, $q-q_{0}$ is the subsidence.

According to relevant theories and experiments, the convergence speed of the simulation is obtained optimally when the soil deformation index $\mathrm{n}$ is ranged from 2 to 3 . The friction between the track and the ground is calculated by Coulomb's law. The parameters of the hard pavement used in the simulation are shown in Table 6.

Table 6. Hard pavement parameters.

\begin{tabular}{cc}
\hline Parameter & Value \\
\hline Road spring coefficient & 10,000 \\
Road damping coefficient & 10 \\
Max friction coefficient & 0.7 \\
Soil deformation index & 2.0 \\
\hline
\end{tabular}

A real-time electronic steering control simulation by the Hardware-in-the-Loop (HIL) method is constructed with rapid simulation prototyping technology, as shown in Figure 7. The Simulink models of electronic differential steering control strategy and motor control strategy are compiled into executable codes via RT-LAB software. Then the executable codes are loaded to RT-LAB hardware 1 and RT-LAB hardware 2 respectively to run the real-time simulation. The required output torque signal of four motors obtained from HIL simulation of ECU are input to RT-LAB hardware 2 for HIL simulation of motors. The output torque signals from HIL simulation of motors are given to the Simulink vehicle model built by Recurdyn. Some typical high-speed steering cases are simulated to verify the feasibility of the coupling device and the strategy proposed.

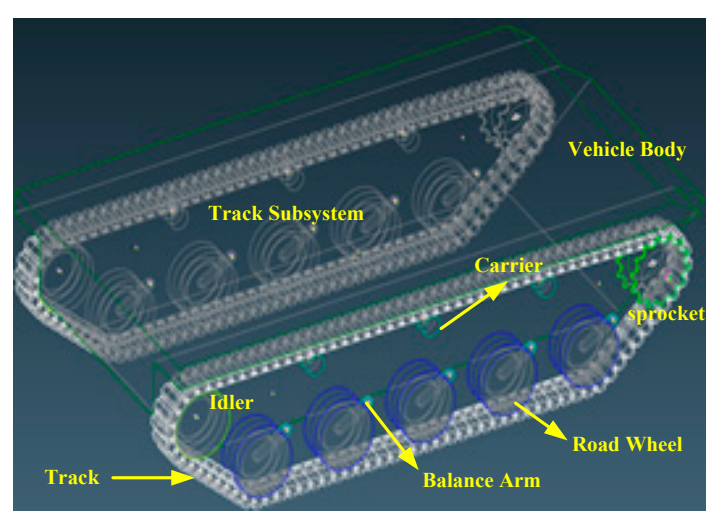

(a)

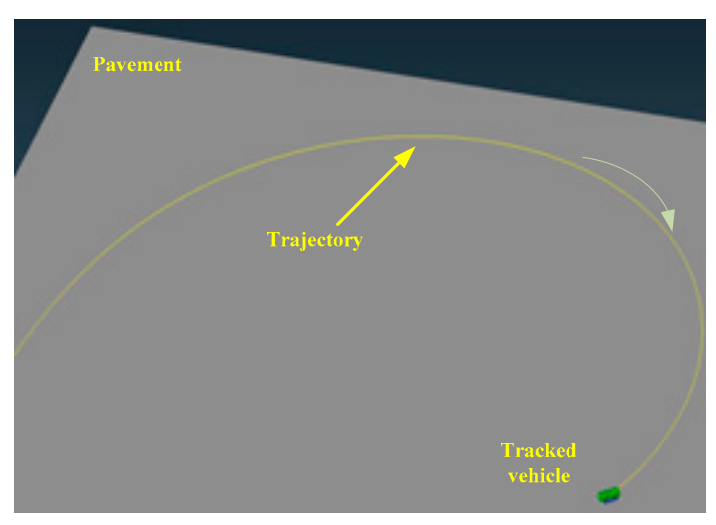

(b)

Figure 6. (a) Vehicle model in RecurDyn; (b) Vehicle model during simulation. 


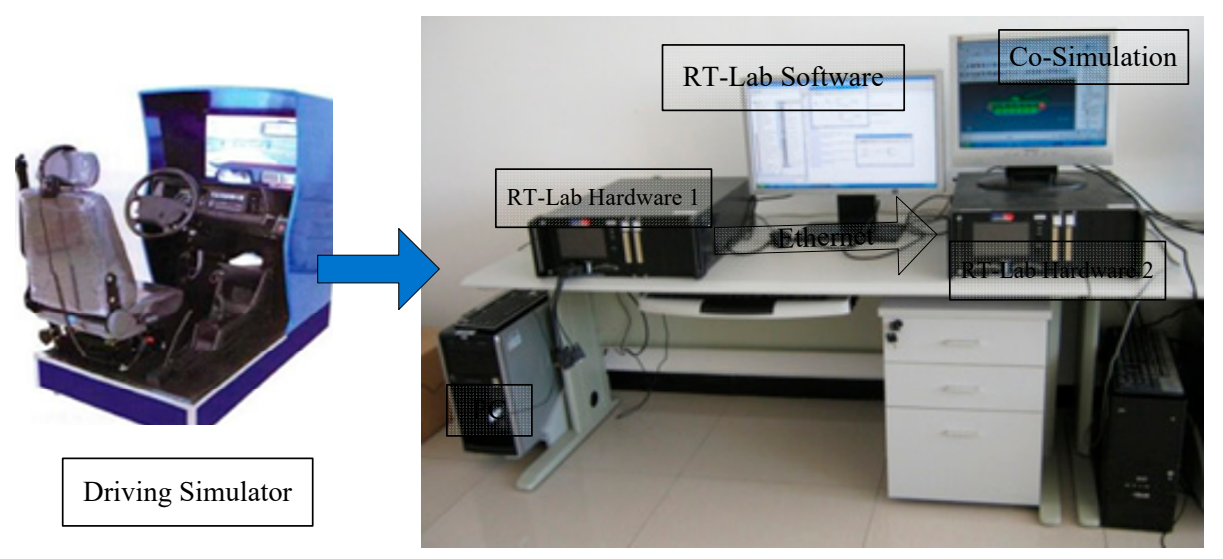

Figure 7. Hardware-in-the-Loop simulation for electronic steering control.

\subsection{B Steering}

At first, the vehicle is stationary for $2 \mathrm{~s}$. After that, the vehicle starts running straight for $5 \mathrm{~s}$ and accelerates to $28.8 \mathrm{~km} / \mathrm{h}$. The steering wheel is turned to the right at $7 \mathrm{~s}$ and manipulated to output $R=5 B$. The trajectory, yaw rate and speed of the vehicle are shown in Figure 8a-c. Without the coupling system, the vehicle speed declines continually, while with the coupling device the vehicle speed is higher, which can maintain near the theoretical value, as shown in Figure 8c. The torque, rotation speed and power of the sprocket are shown in Figure $8 \mathrm{~d}-\mathrm{f}$. Limited by the motor power, the speed of the sprocket is lower without the coupling device. Figure $8 \mathrm{f}$ shows that the output power of outer sprocket is improved from 50 to $60 \mathrm{~kW}$, and the vehicle speed can be maintained with the coupling device. It is worth mentioning that, seen from Figure $8 \mathrm{~b}$, the yaw rate of the vehicle cannot be too large during high speed steering, and otherwise the vehicle is easy to be instability or rollover. Although the yaw rate is lower than what we assumed, but at this situation the power demand is still high (larger than the calculated value).

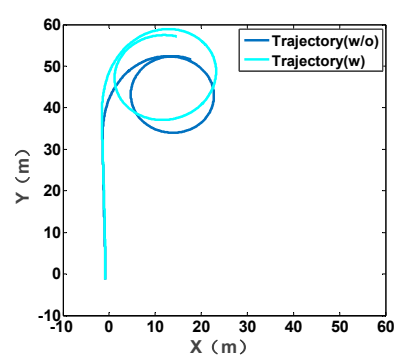

(a)

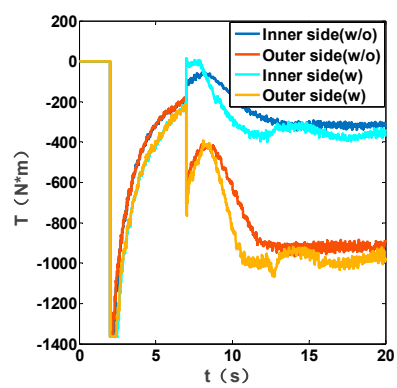

(d)

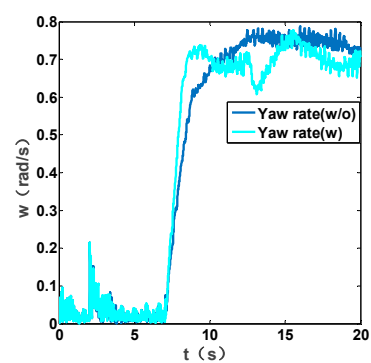

(b)

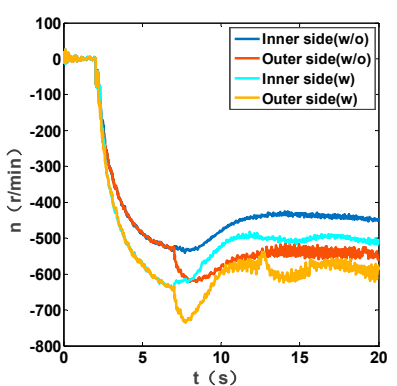

(e)

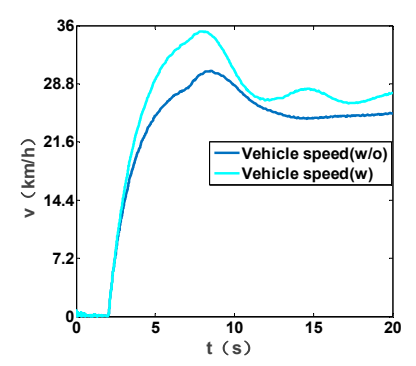

(c)

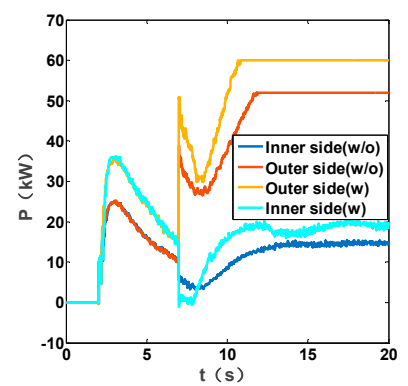

(f)

Figure 8. Simulation results of $5 B$ steering: (a) Trajectory; (b) Vehicle yaw rate; (c) Vehicle speed; (d) Sprocket torque; (e) Sprocket rotation speed; (f) Sprocket power. 


\section{2. $10 B$ Steering}

At first, the vehicle is stationary for $2 \mathrm{~s}$. After that, the vehicle starts running straight for $5 \mathrm{~s}$ and accelerates to $40.7 \mathrm{~km} / \mathrm{h}$. The steering wheel is turned to the right at $7 \mathrm{~s}$ and manipulated to output $R=10 B$. The trajectory, yaw rate and speed of the vehicle are shown in Figure $9 \mathrm{a}-\mathrm{c}$. From Figure $9 \mathrm{~b}$ we know that without the coupling device, the fluctuation of the vehicle yaw rate deteriorate the trajectory controllability, also the vehicle speed fluctuates and declines at the same time. While the speed of the vehicle with coupling device is close to the target value. The torque, rotation speed and power of the sprocket are shown in Figure $9 \mathrm{~d}-\mathrm{f}$. The graph shows that the torque varies smoothly with the coupling device. Without the coupling device, the rotation speed is lower and there are fluctuations due to the limitation of the motor power according to Figure 9e. However, the output power increases with the coupling device, therefore the vehicle can turn in high speed. It is worth noting that the yaw rate is a little lower to ensure the stability of the vehicle, however, the power demand is still quite high especially during dynamic steering.

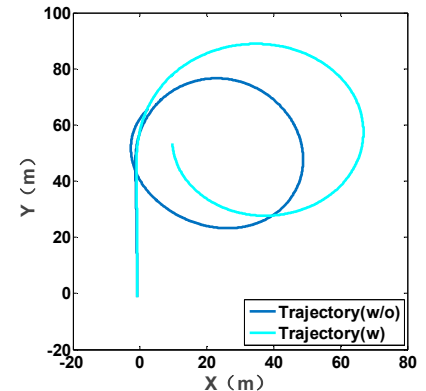

(a)

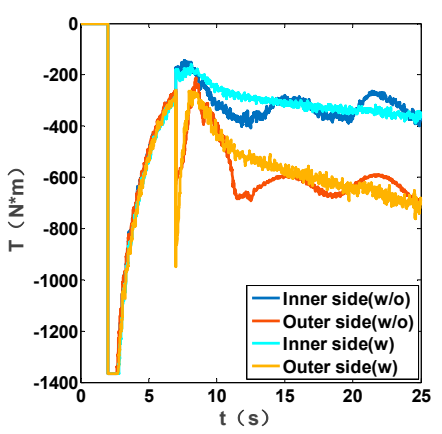

(d)

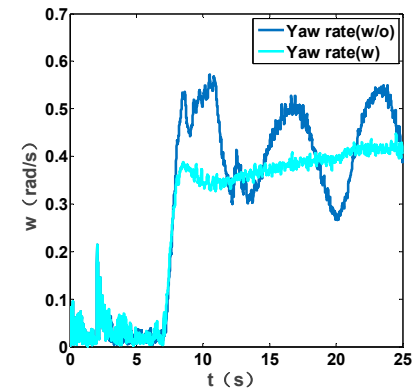

(b)

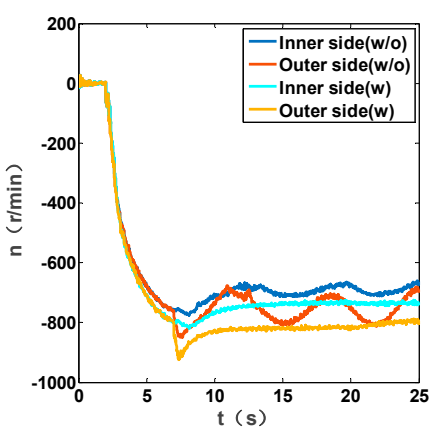

(e)

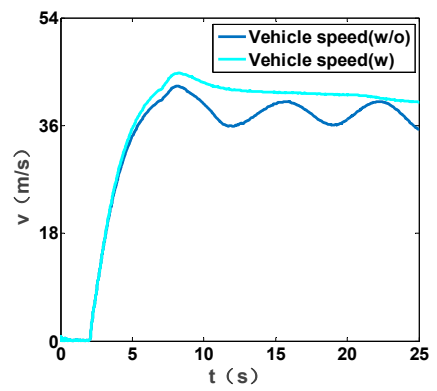

(c)

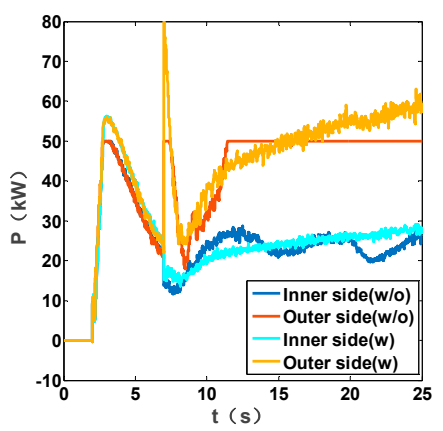

$(\mathbf{f})$

Figure 9. Simulation results of $5 B$ steering: (a) Trajectory; (b) Vehicle yaw rate; (c) Vehicle speed; (d) Sprocket torque; (e) Sprocket rotation speed; (f) Sprocket power.

\subsection{B Steering}

At first, the vehicle is stationary for $2 \mathrm{~s}$. After that, the vehicle starts running straight for $8 \mathrm{~s}$ and accelerates to $57.5 \mathrm{~km} / \mathrm{h}$. The steering wheel is turned to the right at $10 \mathrm{~s}$ and manipulated to output $R=20 B$. The trajectory, yaw rate and speed of the vehicle are shown in Figure 10a-c. We can see from Figure 10a that the steering radius is quite large due to the slip between tracks and the ground during high-speed steering. To avoid the rollover or instability of the vehicle, the yaw rate is not as high as the value we assumed. However, the yaw rate of the vehicle with coupling device is still higher. The vehicle speed is higher with the coupling device while $11 \%$ lower than the theoretical value according to Figure 10c. The torque, rotation speed and power of the sprocket are shown in Figure 10d-f. It can be seen that the output torque and the speed of the sprocket are not changed greatly during stationary steering due to the limitation of the motor power. The output torque and the speed of the sprocket is lower without the coupling device. While with the coupling device the 
output power is improved and the vehicle speed can be maintained at higher level. Although the yaw rate is not large, the power demand is still larger than the calculated value to maintain the high speed during steering.

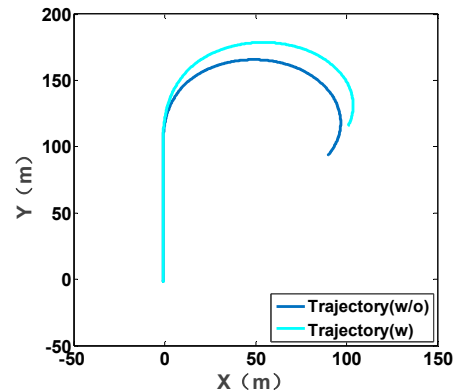

(a)

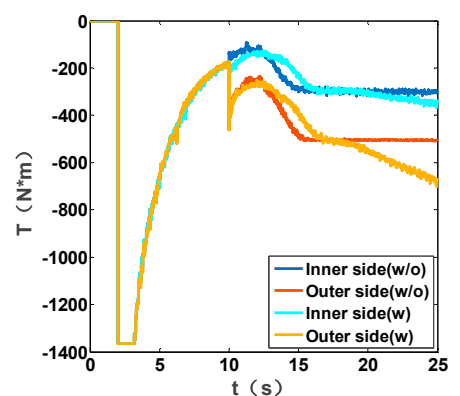

(d)

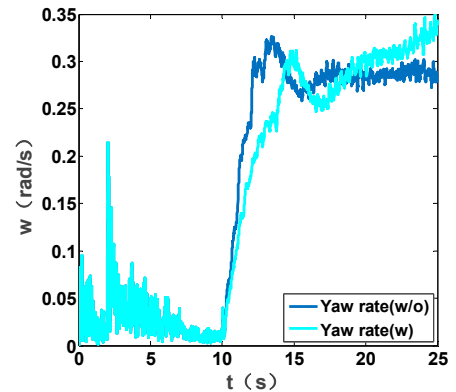

(b)

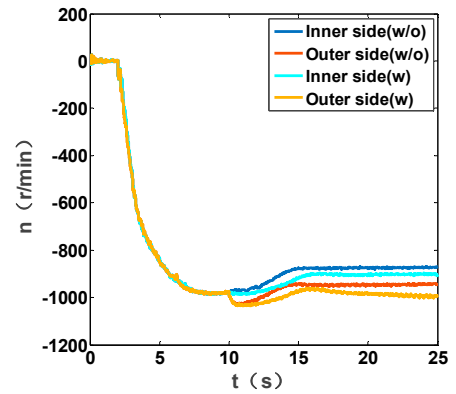

(e)

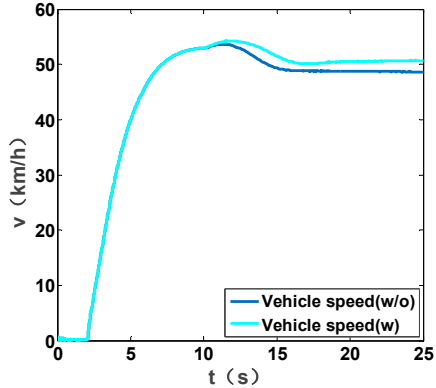

(c)

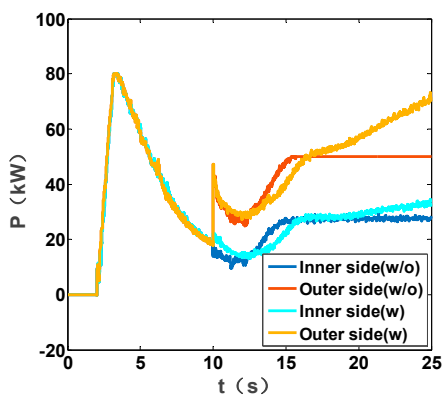

(f)

Figure 10. Simulation results of $5 B$ steering: (a) Trajectory; (b) Vehicle yaw rate; (c) Vehicle speed; (d) Sprocket torque; (e) Sprocket rotation speed; (f) Sprocket power.

\section{Conclusions}

In this paper, according to the dynamic analysis of the 2MDTV, we found that the power required by the propulsion motor is quite large during high-speed steering situations. As the existing research focused more on low-speed conditions, we considered the vehicle performance during high speed steering in this work and supplemented the calculations about the power demand. In order to solve the problem of understeer and speed decline of the vehicle caused by insufficient power during high speed steering, a new electromechanical coupling device is proposed. The device is used to couple the power of the steering motor and the propulsion motor to satisfy the requirements for high-speed steering. The coupling system can increase the power of outer track by $20 \mathrm{~kW}$ during high-speed steering. Furthermore, an optimized torque distribution control strategy is proposed. In the Hardware-in-the-Loop (HIL) simulation of 5B, 10B, and $20 B$ steering with RecurDyn and Matlab/Simulink models, the coupling device can improve the output power of the vehicle, and the proposed strategy can improve the steering stability and achieve smoother steering response of the vehicle at high speed.

Author Contributions: L.Z. proposed the innovation of the overall system and designed the control methods, H.H. are responsible for the improvement of coupled systems and the simulation of the system, Z.W. and H.H. built the mathematic model and performed the calculation. L.Z. and H.H. wrote the manuscript and Z.W. polished the manuscript. All authors read and approved the manuscript.

Acknowledgments: This work was supported by the National Natural Science Foundation of China for financially supporting this project (51475045).

Conflicts of Interest: The authors declare no conflict of interest. 


\section{References}

1. Yan, N. Electric drive systems in armored tracklayer vehicles. Acta Armamentarii 2004, 25, 619-623.

2. Sun, F.C.; Zhang, C.N. Technologies for the Hybrid Electric Drive System of Armored Vehicles, 2nd ed.; National Defense Industry Press: Beijing, China, 2016.

3. Luo, H.W.; Chen, Y.D.; Yang, Z.H.; Sun, G.; Ye, L.G.; Liu, H.B.; Cui, S.G.; Chen, S.K.; Zhang, H.L.; $\mathrm{Xie}, \mathrm{W}$; et al. Steering differential control of electric transmission tracked vehicles based on fuzzy modeling. Control Eng. China 2016, 23, 1515-1518.

4. Zhai, L.; Sun, T.M.; Wang, Q.N.; Wang, J. Lateral stability control of dynamic steering for dual motor drive high speed tracked vehicle. Int. J. Autom. Technol. 2016, 17, 1079-1090. [CrossRef]

5. Liu, L. Study on Power Requirements Dynamic Characteristics of Dual-motor Drive Motor Tracked Vehicle. Master's Thesis, Beijing Institute of Technology, Beijing, China, 2012.

6. Jia, X.P.; Ma, J.; Yu, K.L.; Li, J. Performance Analysis of the Electric Tracked Vehicle Coupling Transmission Scheme. J. Mech. Transm. 2015, 39, 129-132.

7. Jia, X.P.; Jun, M.A.; Fan, S.G.; Yu, K.-L. Simulation of coupling mechanism for electric-drive off-road vehicle. Veh. Power Technol. 2014, 136, 6-10.

8. Jia, X.P.; Ma, J.; Yu, K.L. Study on Transmission System Scheme of Electric Drive Tracked Vehicles. J. Acad. Armored Force Eng. 2015, 2, 35-39.

9. Gai, J.; Huang, S.; Zhou, G.; Li, S. Design method of power coupling mechanism scheme for double side motors coupling drive transmission. China Mech. Eng. 2014, 13, 1739-1743.

10. Control Differential Transmission Gearing. U.S. Patent 2,730,182, 10 October 1956.

11. Wang, W. Integrated control strategy of electro-mechanical transmission system. J. Mech. Eng. 2011, 47, 152-158. [CrossRef]

12. Dong, Y.G.; Zhang, C.N.; Wu, X.H. A Dynamic Coupling System for Main and Auxiliary Motors of Dual Motor Independent Drive Vehicle. CN 102133855A, 27 July 2011.

13. Zhai, L.; Wang, Q.N.; Liu, L. Steering System with Coupling of Electric Drive Tracked Vehicle Steering Motor and Unilateral Drive Motor. CN 102700611 B, 11 March 2015.

14. Yuan, Z.; Sun, F.C.; Zhang, C.N. Dual-motor driving electric tracked vehicle speed-regulating control strategy. Trans. Beijing Inst. Technol. 2007, 27, 303-307.

15. Yuan, Z.; Sun, F.C.; Zhang, C.N. Torque-regulating control strategy of electric tracked vehicle driven by dual-motor. Acta Armamentarii 2007, 28, 1409-1414.

16. Zeng, Q.H.; Xiao-Jun, M.A.; Wei, W.; Dong, Y. Research on coordination control strategy of driving force of distributed electric drive tracked vehicle. Acta Armamentarii 2017, 38, 9-19.

17. Liu, Y.; Gai, J.T.; Chen, Y.D.; Wan, F. Self- adaptive steering control of electric drive for tracked vehicles. Veh. Power Technol. 2015, 1, 5-10.

18. Wang, H.; Huang, Y.; Khajepour, A.; He, H.; Cao, D. A Novel Energy Management for Hybrid Off-road Vehicles without Future Driving cycles as A Priori. Energy 2017, 133, 929-940. [CrossRef]

19. Zou, Y.; Kong, Z.; Liu, T.; Liu, D. A Real-Time Markov Chain Driver Model for Tracked Vehicles and Its Validation: Its Adaptability via Stochastic Dynamic Programming. IEEE Trans. Veh. Technol. 2017, 66, 3571-3582. [CrossRef]

20. Wei, S.; Zou, Y.; Sun, F.; Christopher, O. A pseudospectral method for solving optimal control problem of a hybrid tracked vehicle. Appl. Energy 2017, 194, 588-595. [CrossRef]

21. Liu, T.; Zou, Y.; Liu, D.; Sun, F. Reinforcement learning-based energy management strategy for a hybrid electric tracked vehicle. Energies 2015, 8, 7243-7260. [CrossRef]

22. Zhai, L.; Huang, H.; Sun, T.; Wang, Q. Investigation of energy efficient power coupling steering system for dual motors drive high speed tracked vehicle. Energy Procedia 2016, 104, 372-377. [CrossRef]

23. Zhai, L.; Huang, H.; Kavuma, S.; Sciubba, E. Investigation on a power coupling steering system for dual-motor drive tracked vehicles based on speed control. Energies 2017, 10, 1118. [CrossRef]

24. Ma, B.; Qian, S. Analysis of minimum rolling resistance factor $\mathrm{f}(\mathrm{min})$ and moving system efficiency $\eta \mathrm{x}$ of tracked vehicles. Acta Armamentarii 1999, 1, 44-48.

25. Bekker, M.G. Introduction to Terrain-Vehicle Systems; University of Michigan Press: Ann Arbor, MI, USA, 1969. 
26. Yan, Q.D.; Zhang, L.D.; Zhao, Y.Q. Tank Structure and Design; Beijing Institute of Technology Press: Beijing, China, 2006.

27. Liu, B.; Wang, Z.F. Overview of power system development of tracked vehicle. J. Sichuan Ordnance 2014, $35,68-72$. 